\title{
Hybrid of Graphitic Carbon Nitride and Palladated Magnetic Carbon Dot: An Efficient Catalyst for Coupling Reaction
}

\author{
Leila Mohammadi ${ }^{1}, \underline{\text { Majid M. Heravi }}{ }^{1}$, Samahe Sadjadi $^{2}$, Masoumeh Malmir ${ }^{1}$ \\ 1- Department of Chemistry, School of Science, Alzahra University, Vanak, Tehran, PO Box \\ 1993891176, Iran \\ 2- Gas Conversion Department, Faculty of Petrochemicals, Iran Polymer and Petrochemicals \\ Institute, Tehran, PO Box 14975-112, Iran
}

To take advantage of the properties of graphitic carbon nitrides and carbon quantum dots, a novel hybrid system was designed based on the synthesis and palladating of magnetic carbon quantum dots followed by hydrothermal treatment with graphitic carbon nitrides. The resulting hybrid, g-C3N4Pd/CQDs@Fe, was then utilized as an efficient catalyst for promoting ligand and co-catalyst-free Sonogashira coupling reaction under mild reaction condition in aqueous media. Not only the catalyst could catalyze the reaction of broad range of the substrates, but also it showed good recyclability and could be easily recovered by using an external magnet and reused for several times. The study of the catalytic activities of palladated graphitic carbon nitride and magnetic carbon quantum dots confirmed that the hybrid system was more efficient catalyst compared to each component. 